\title{
Computer-Assisted High Tibial and Double Level Osteotomies for Genu Varum Deformity
}

\author{
Dominique Saragaglia ${ }^{1}$ and Sam Hakki ${ }^{2}$ \\ ${ }^{1}$ Department of Orthopaedic Surgery and Sport Traumatology, \\ Grenoble South Teaching Hospital, Échirolles, \\ ${ }^{2}$ Bay Pines Health Care System Hospital, St Petersburg, Florida \\ ${ }^{1}$ France \\ ${ }^{2} U S A$
}

\section{Introduction}

Medial compartment knee osteoarthritis is not uncommon and high tibial osteotomy (HTO) was described for the first time more than 50 years ago (Jackson, JP \& Waugh, W 1961; Judet, R \&Dupuis, JF \& Honnard, F 1964; Merle d'Aubigné, R \& Ramadier, JO 1961). Nowadays, it remains a good option (Coventry, MB \& Ilstrup, DM \& Wallrichs, SL 1993; Hernigou, Ph \& Medevielle, D \& Debeyre, J 1987; Jenny JY \& Tavan, A \& Jenny, G, et al 1998; Lerat, JL 2000; Lootvoet, L \& Massinon, A \& Rossillon, R, et al 1993; Papachristou, G \& Pleassa, S \& Sourlas, J \& Levidiotis, C \& Chronopoulos, E \& Papachristou, C 2006; Rinonapoli, E \& Mancini, GB \& Corvaglia, A, et al 1998; Saragaglia, D \& Blaysat, M \& Inman, D \& Mercier, N 2010; Yasuda, K \& Majima, T \& Tsuchida, T, et al 1992) despite the large expansion of total knee replacement (TKR) or the revival of unicompartmental knee prosthesis boosted by the less-invasive surgery concept. Ideally, HTO is indicated for active patients who are less than 65 years of age with moderate arthritis (narrowing joint line up to $100 \%$ without any bone wear or joint instability). Nevertheless, special attention should be paid to joint line orientation which can be distorted by excessive over or under correction which will eventually lead to earlier failure (Saragaglia, D \& Blaysat, M \& Inman, D \& Mercier, N 2010) due to obliquity of joint line (Fig.1). This oblique joint line corresponds to an excessive valgus of the tibial mechanical axis (Babis, GC \& An, KN \& Chao, E. YS, et al 2002). Moreover, varus knee deformity may be a result of both tibial and femoral deformities. Correction of combined (femoral and tibial) varus deformity at the tibia level by $\left(3^{\circ}\right.$ to $\left.6^{\circ}\right)$ to achieve a good clinical result may worsen the obliquity of knee joint line.

We have considered combined femoral and tibial osteotomy as a solution to avoid excessive joint line obliquity. However, prior to the advent of computer navigation this was only performed on a limited basis because of the difficulty in obtaining an accurate mechanical axis in relation to joint line plane.

Drawing on our experience with TKR and HTO navigation (Picard, F \& Leitner, F \& Raoult, A, et al 1999; Saragaglia, D \& Pradel, Ph \& Picard, F 2004; Saragaglia, D \& Picard, F \& 
Chaussard, et al 2001; Saragaglia, D \& Roberts, J 2005; Saragaglia, D \& Rubens-Duval, B \& Chaussard, C 2007; Hakki, S \& Saleh, K \& Bilotta, V et al) we used the principles of computerassisted surgery for double level osteotomy (DLO) hoping to increase the accuracy of this difficult procedure. Our experience is based on 42 DLO performed between August 2001 and June 2010, out of 370 computer-assisted knee osteotomies for genu varum deformities $(11.3 \%)$.We will present first, the preoperative radiological assessment, the computer-assisted operative procedure and the indications of HTO, DLO and distal femoral osteotomy (DFO). Then we will present the rationale behind this way of thinking and our results.

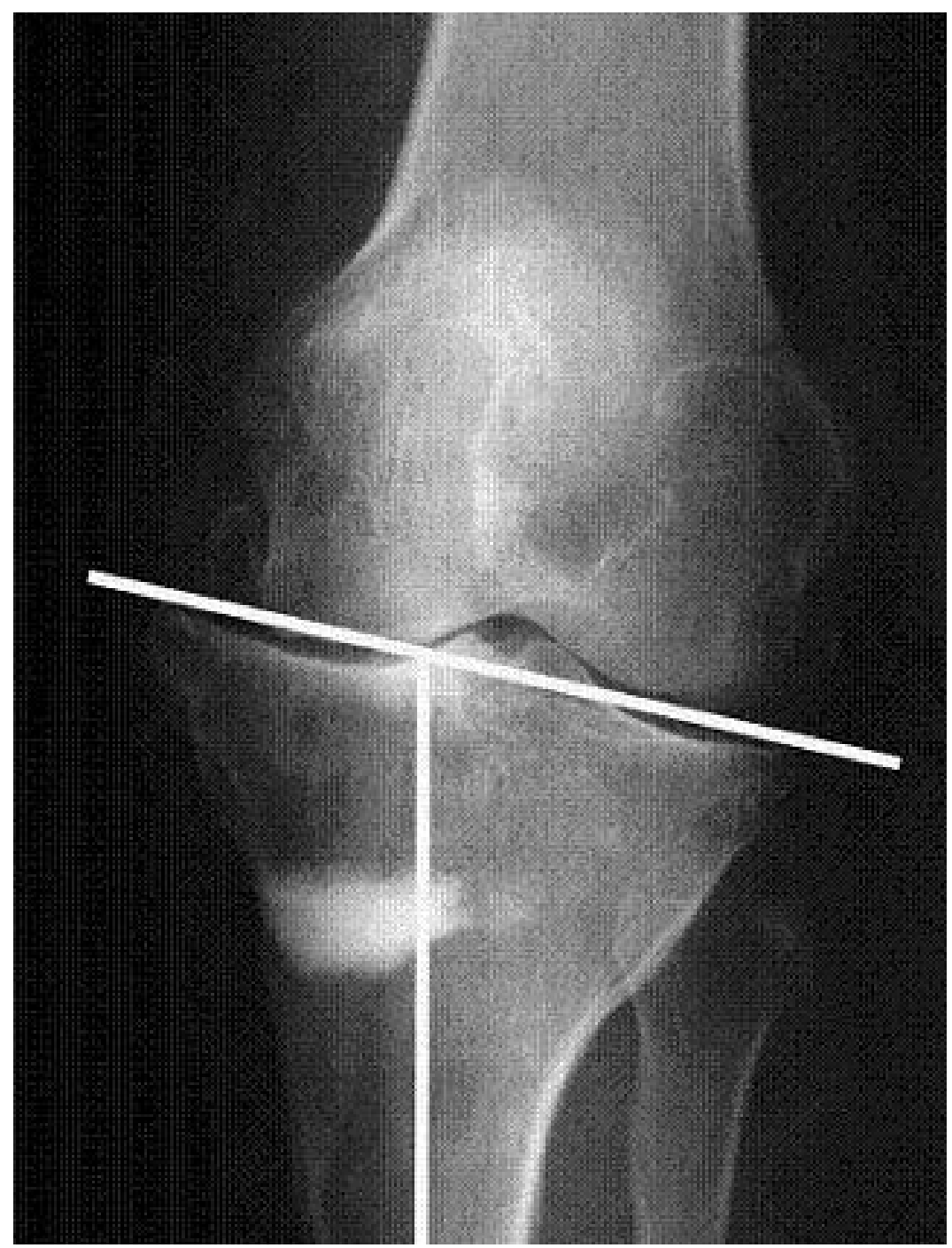

Fig. 1. Although varus deformity of the left knee was corrected the joint line remained in excessive valgus. This will lead to early failure HTO in this case. 


\section{Radiological assessment}

Preoperatively Standing AP, lateral, and 45 degree PA weight bearing (Rosenberg) views are obtained. In addition, it is essential to obtain AP long leg standing $\mathrm{x}$-rays to assess the hip knee ankle (HKA) angle for preoperative planning. Ramadier's protocol (Ramadier, JO \& Buard, JE \& Lortat-Jacob, A, et al 1982) allows these measurements to be reproducible pre and postoperatively. This protocol can be described as follows: first, to determine accurately the frontal plane by looking for a true lateral view of the knee which is obtained when the posterior margins of the condyles are superimposed; secondly, to turn $90^{\circ}$ around the knee the image intensifier to obtain an accurate long leg AP standing view, the $x$-ray being perpendicular to the frontal plane; finally, to draw the foot print on a cardboard in order to reproduce the same rotation of the lower leg pre and postoperatively. Using this cardboard by placing the foot in the print, it is easy to do the same view as much as one wants. The long leg film is critical since the deformity may not be visible on standard knee films (Fig. 2a and $2 b$ ). One must measure the HKA angle, the medial distal femoral mechanical angle (MDFMA) and the medial proximal tibial mechanical angle (MPTMA)(Fig. 3a and 3b) in order to plan the level of the osteotomy femoral, tibial, or both. (Fig 3c)

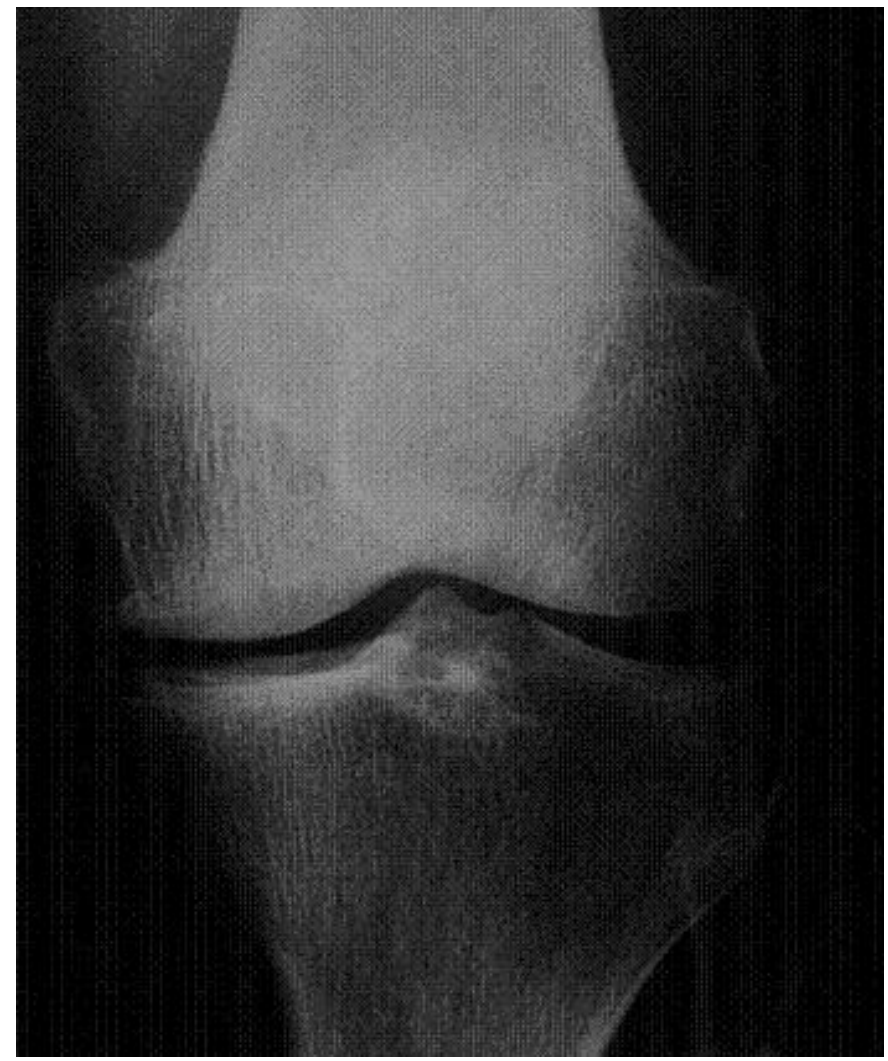

Fig. 2. a: Standard non weight bearing knee films does not show the significant varus deformity this patient has. 


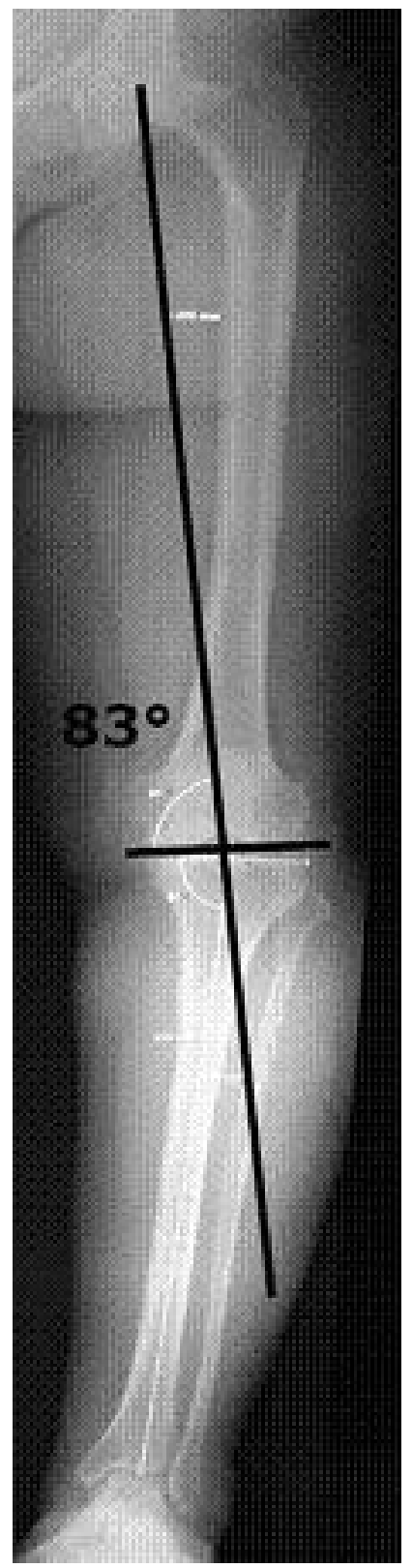

Fig. 2. b: The weight bearing long films serve many purposes: 1 - It shows significant varus deformity in comparison to figure 2a of the same patient. 2 - Quantitative measurements can be made of the severity of varus deformities in relation to the mechanical axis.

3 - Determines where the joint line is. 4 - Quantifies the deformity whether its femoral, tibial, or both. 


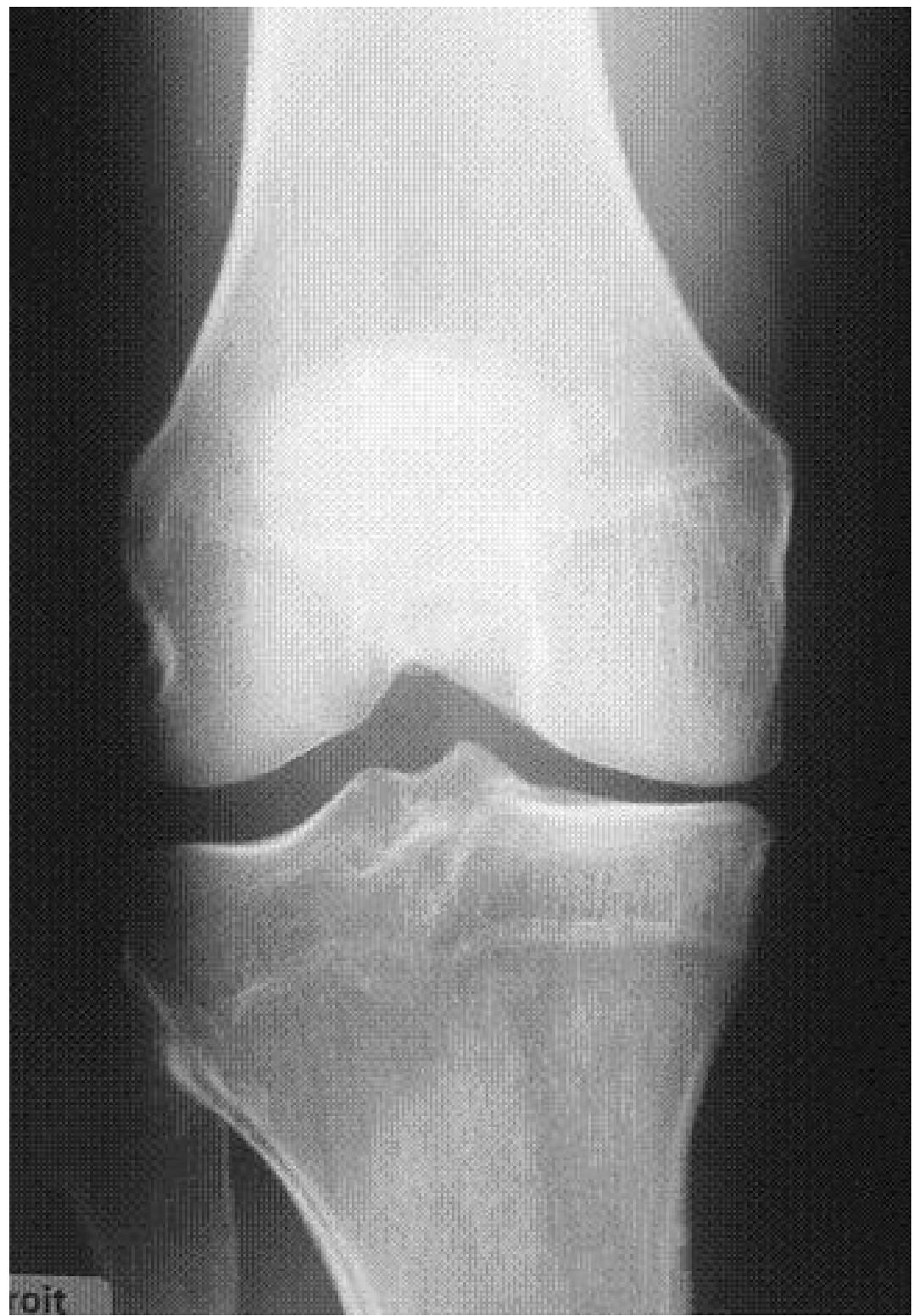

Fig. 3. a: 42 year old female with what seems to be mild tibia varus deformity

Grading of osteoarthritis is performed typically using the modified Ahlbäck classification (Saragaglia, D, Roberts, J 2005) (grade I, < 50\% joint space narrowing; grade II, 50-100\%; grade III, $100 \%$ narrowing without any bone wear; grade IV, bone wear but no lateral instability; grade $\mathrm{V}$, bone wear with lateral compartment degeneration with or without posterolateral subluxation). 


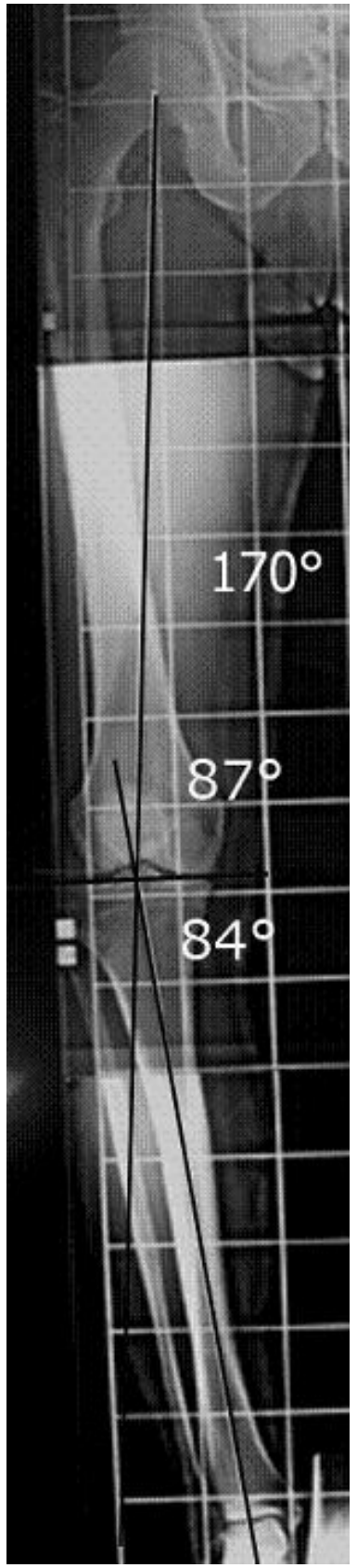

Fig. 3. b: Pre-operative long weight bearing $x$-ray in the same patient as $3 a$ showing $6^{0}$ of femoral and $6^{0}$ of tibial varus deformity. Correction of one bone will not restore the joint line. 


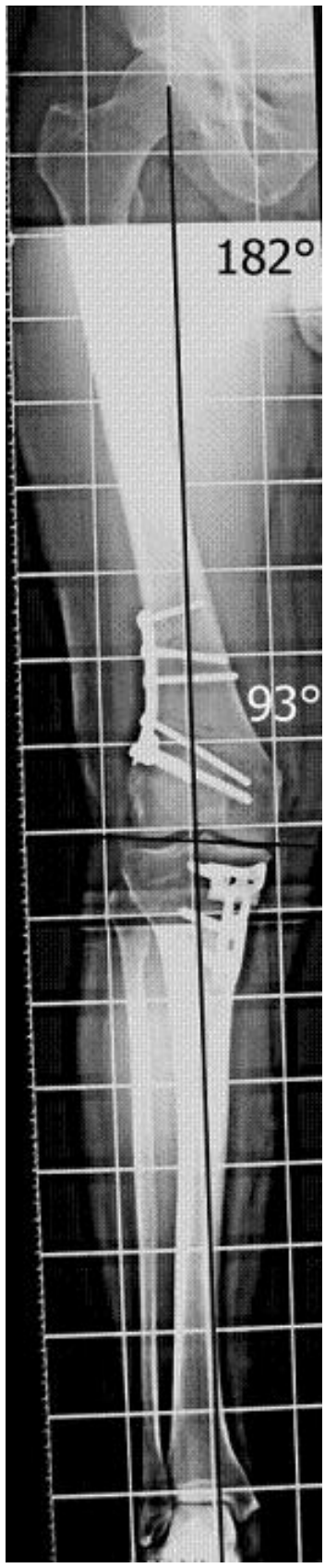

Fig. 3. c: Post operative $x$-ray film showing correction of both femoral and tibial varus deformity with restoration of the joint line and correction of varus deformity to a $2^{0}-3^{0}$ valgus angle. 


\section{Surgical technique}

\subsection{Opening wedge HTO computer-navigated}

The software is a derivative of the one used for TKA which has been fully described elsewhere (Picard, F \& Leitner, F \& Raoult, A etal 1999; Hakki, S \& Saleh, K \& Bilotta, V, et al.) (Orthopilot ${ }^{\circledR}$ Navigation System, B-Braun-Aesculap, Tuttlingen, Germany). The same principal of real time acquisition of the rotation centre of the hip, knee and ankle centres and of the anatomical landmarks at the level of the knee joint line and ankle is applied. They allow the mechanical axis of the lower limb to be shown dynamically on the computer screen, i.e. the axis of the lower limb to be seen both pre and post osteotomy and to check if the pre-planned correction has been established.

The rigid body markers are fixed percutaneously at the level of the distal femur and proximal tibia allowing acquisition of the centres of the hip, knee and ankle (Fig.4). The lower limb mechanical axis then appears on the screen and can be compared with the preoperative radiological goniometry.

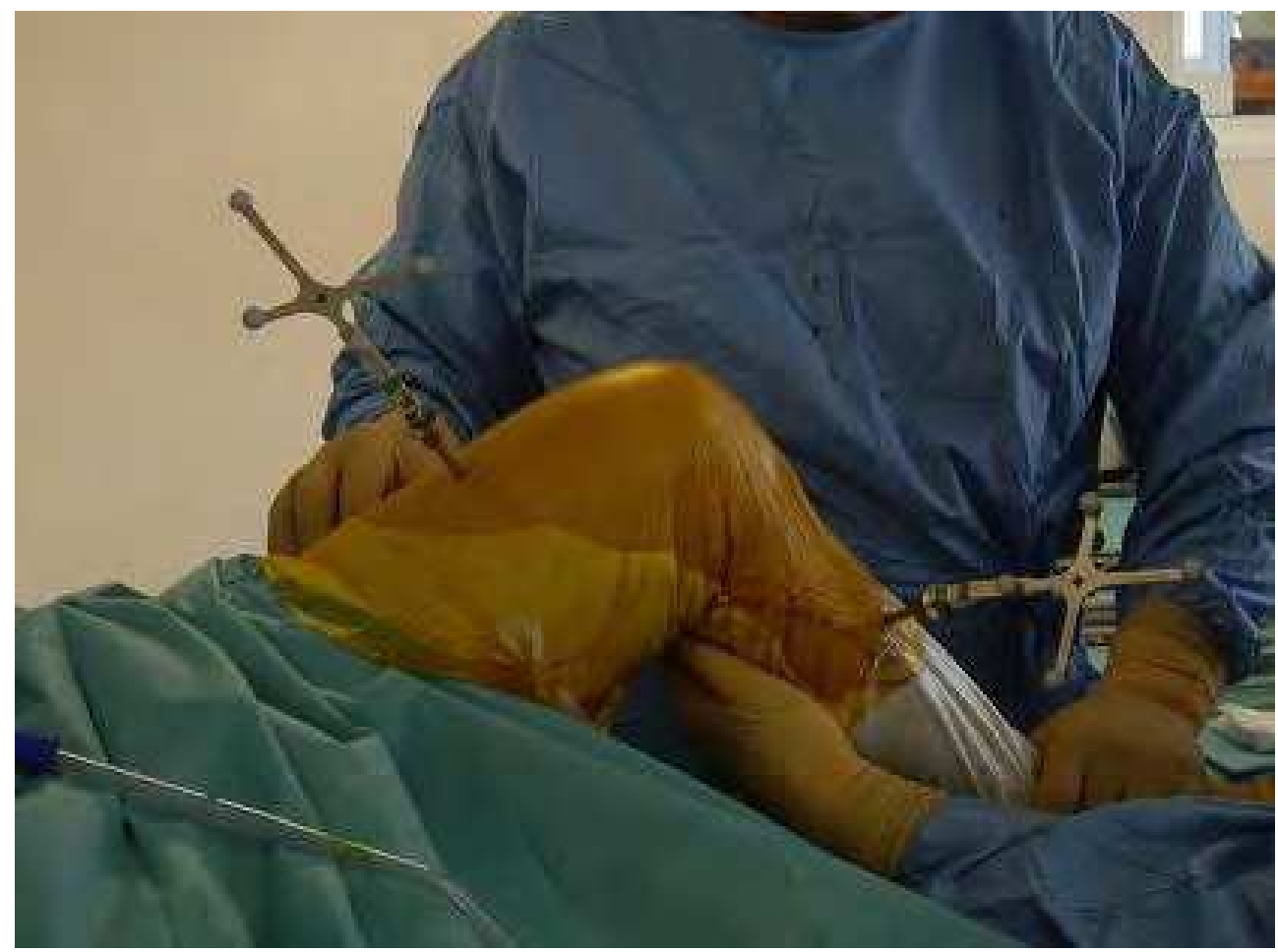

Fig. 4. Computer navigation is used to accurately quantify the deformity in all range of motion of the left knee.

A 5 to 6 centimeter long incision is made on the medial upper end of the tibia just at the level of the anterior tuberosity of the tibia. The pes anserinus is incised just above the gracilis tendon and a retractor is placed against the postero medial corner of the tibia (Fig.5). 
Then, the superficial medial collateral ligament is released from its tibial insertion to allow an adequate opening of the osteotomy.

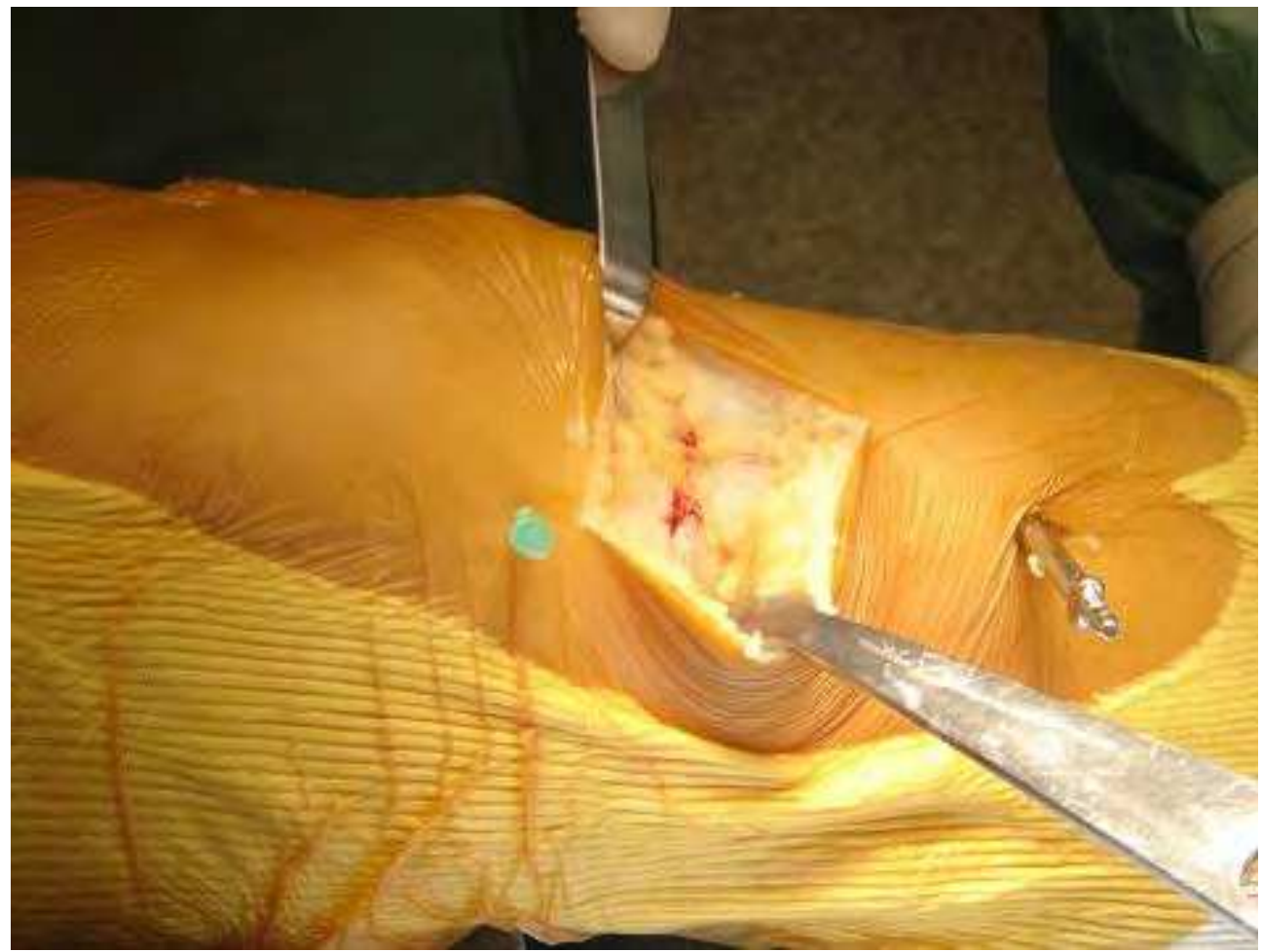

Fig. 5. Incision is made proximal to the tracker of the tibia at the level of tibia tuberosity. Note: pes anserinus is incised just above the gracilis tendon.

The HTO is then performed $3 \mathrm{~cm}$ below the level of the medial joint line, the level of which is confirmed by placing an intra-articular needle. The osteotomy is directed at the fibula head, keeping the saw as horizontal as possible to avoid fracturing the lateral tibial plateau. With the aid of 2 Pauwels osteotomes inserted along the tract of the saw cut, the tibia is placed into valgus (Fig.6).

These are then replaced by a metal spacer, which is inherently stable and allows the amount of correction to be accurately checked. If there was $8^{\circ}$ of varus one would try a $10-11 \mathrm{~mm}$ spacer and check to make sure an appropriate hypercorrection is produced real time on the computer screen (Fig. 7). If this is insufficient we try a thicker spacer and the reverse if the correction is too great.

The metallic spacer is then replaced with a bio-absorbable $\beta$ Tricalcium phosphate wedge (Biosorb ${ }^{\circledR R}$, SBM, Lourdes, France) of the desired thickness (Fig.8), and the intervention completed by plating the proximal tibia (Fig.9 and 10 a,b,c). Then the accuracy of the osteosynthesis is checked with the image intensifier and the wound is closed. 


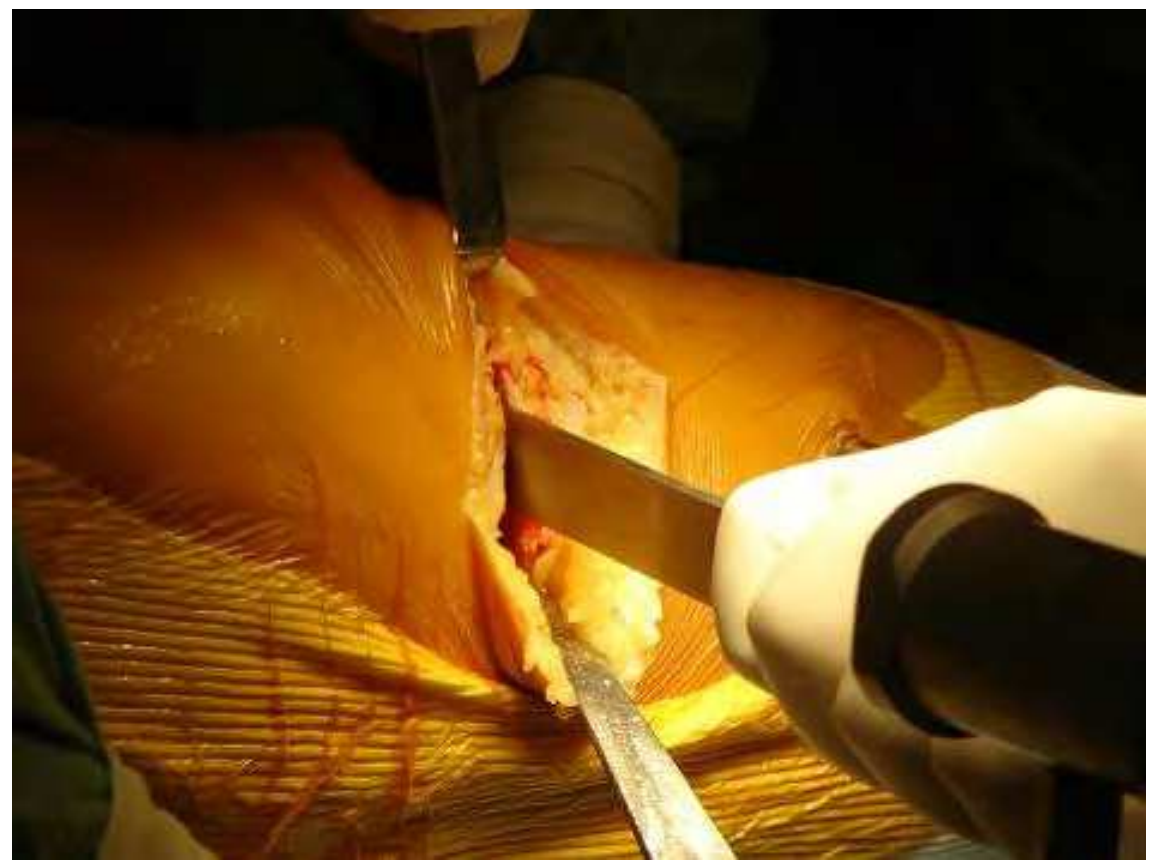

Fig. 6. The superficial part of medial collateral ligament is released to allow opening of the wedge osteotomy.

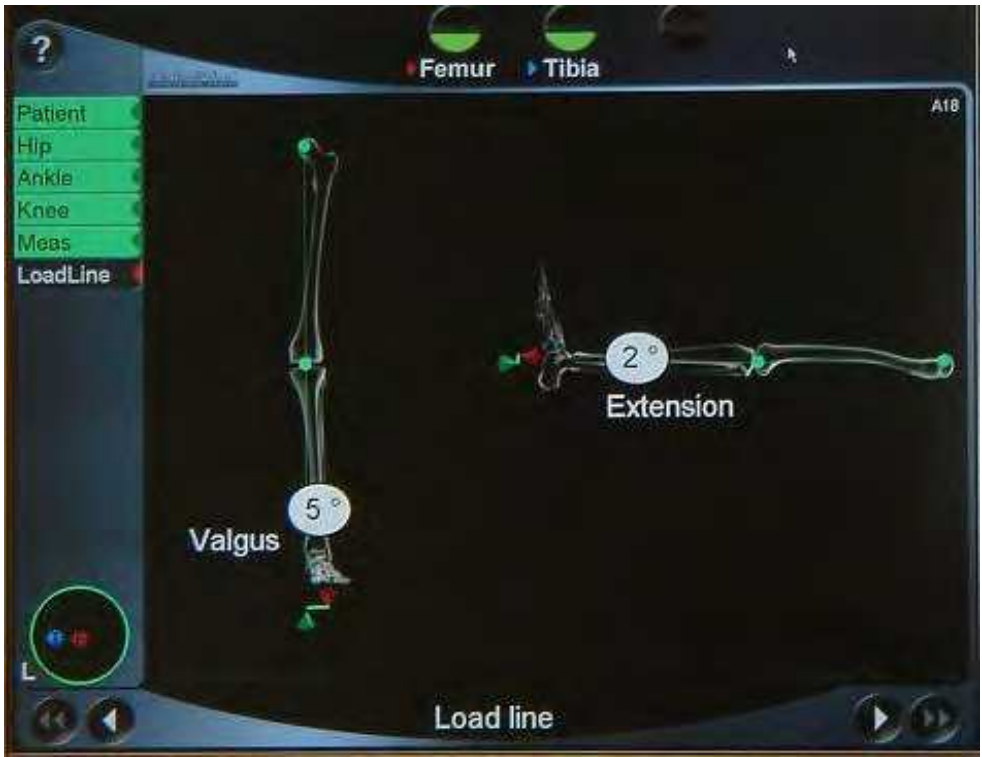

Fig. 7. The real time, exact degree of varus correction is shown on the screen. This will help surgeons to avoid over or under correction of varus deformities. 


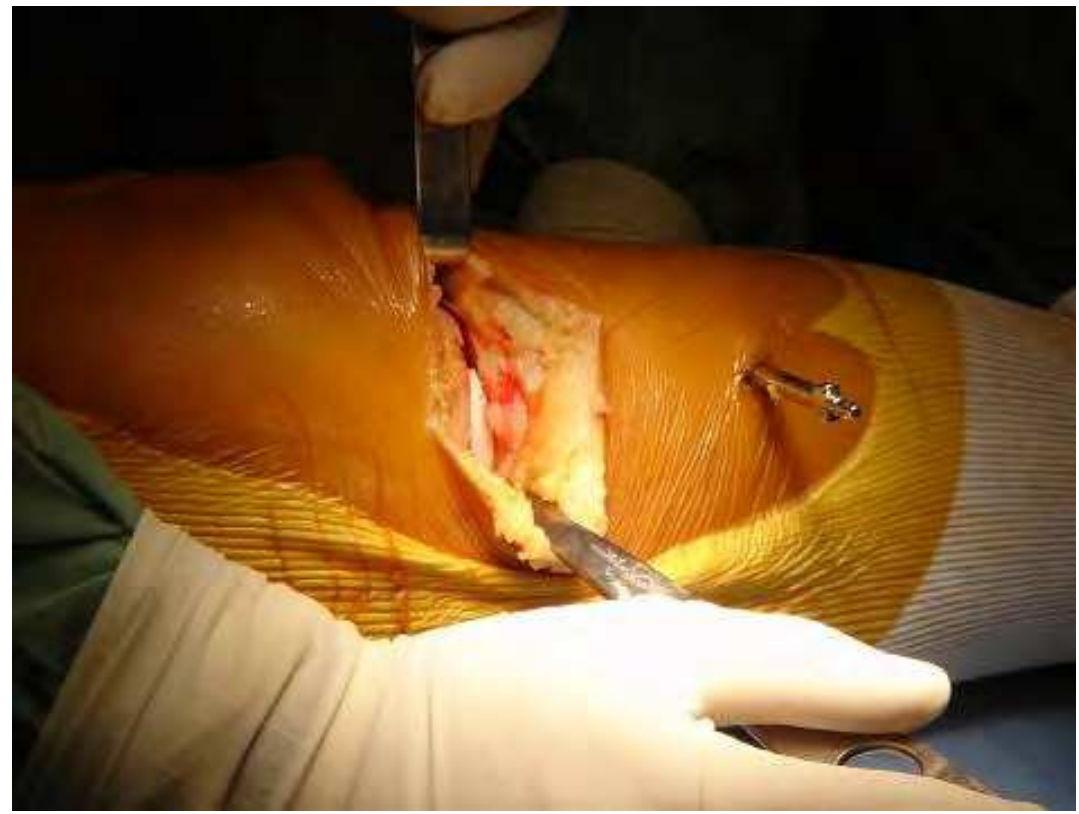

Fig. 8. The exact size of bio-absorbable spacer is determined by computer navigation reading.

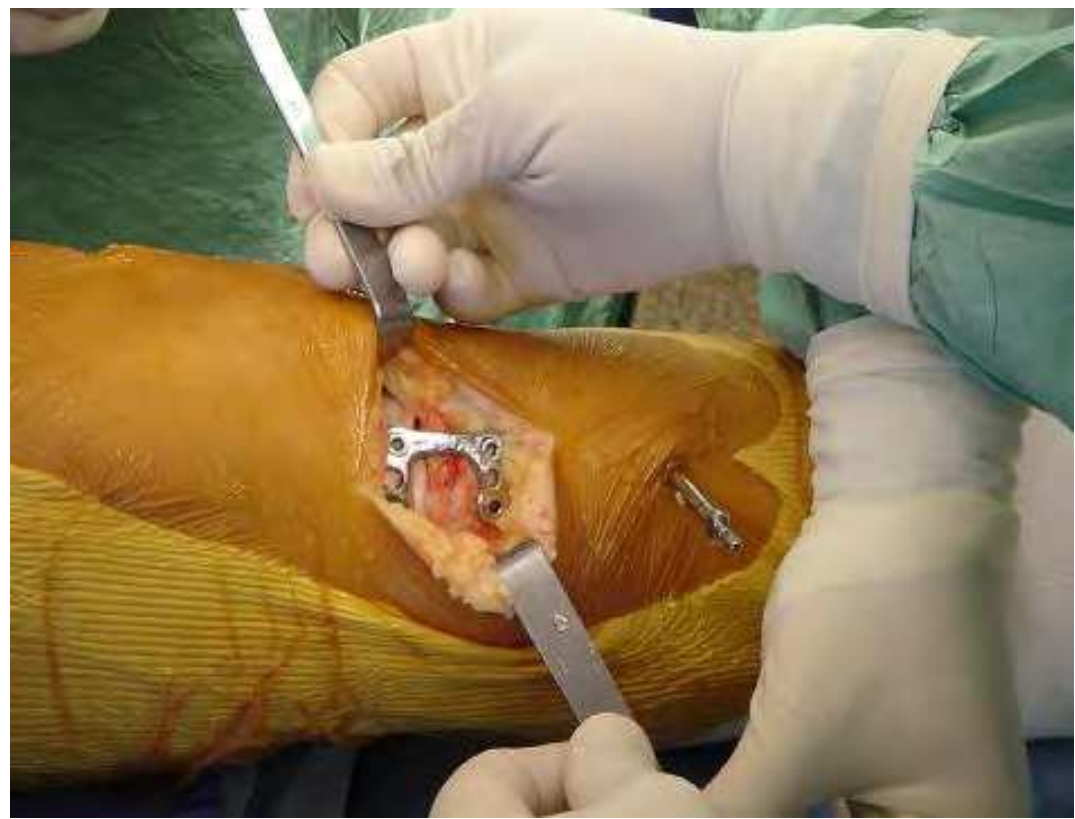

Fig. 9. Plate and screws are used to maintain the correction at the desired angle as determined by the computer. Long film Röntgenogram may also be used to confirm alignment. 


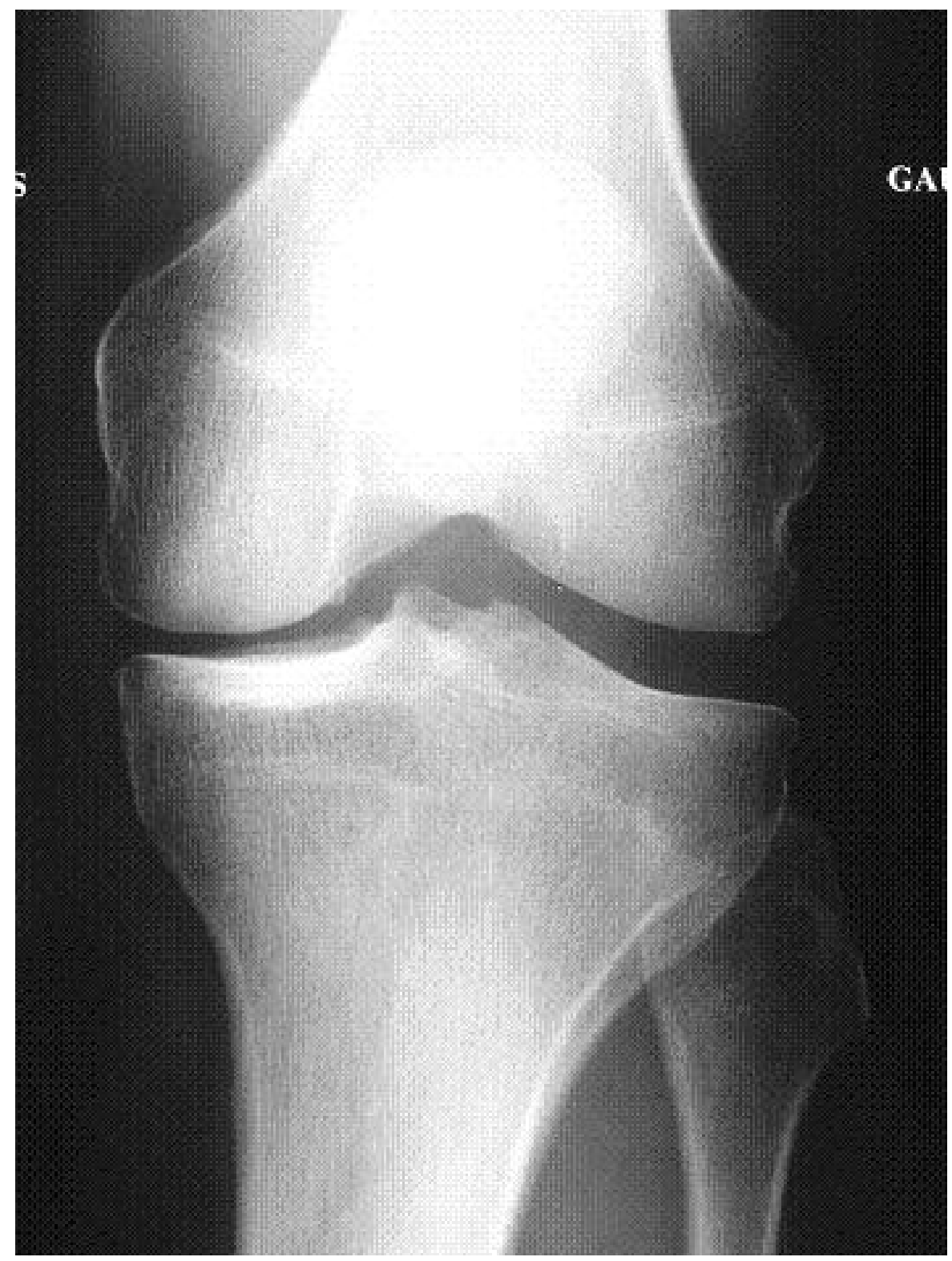

Fig. 10. a: Preoperative standard left knee film showing varus deformity of left knee 


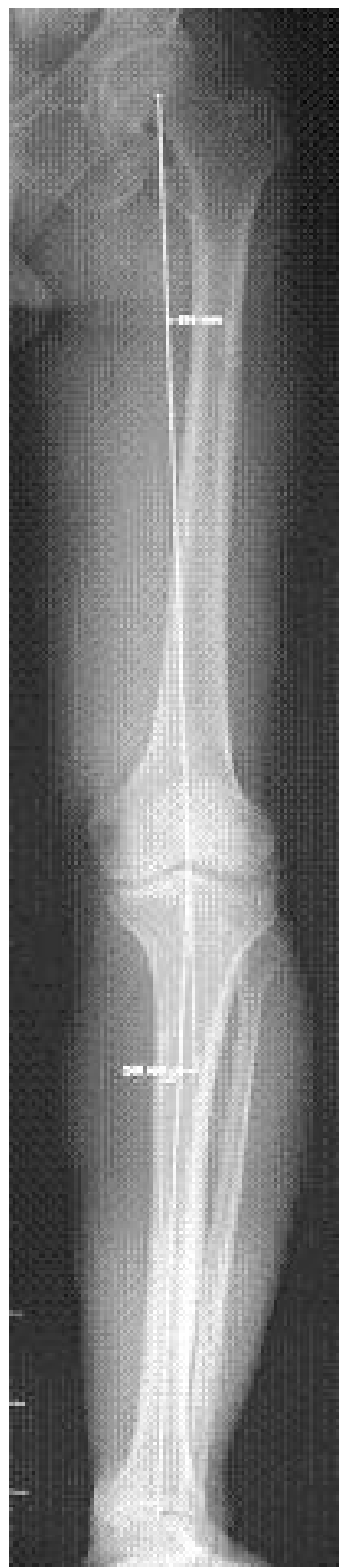

Fig. 10. b: Weight bearing long film determined that the varus deformity is only in the tibia. Joint line and femur were anatomical. 


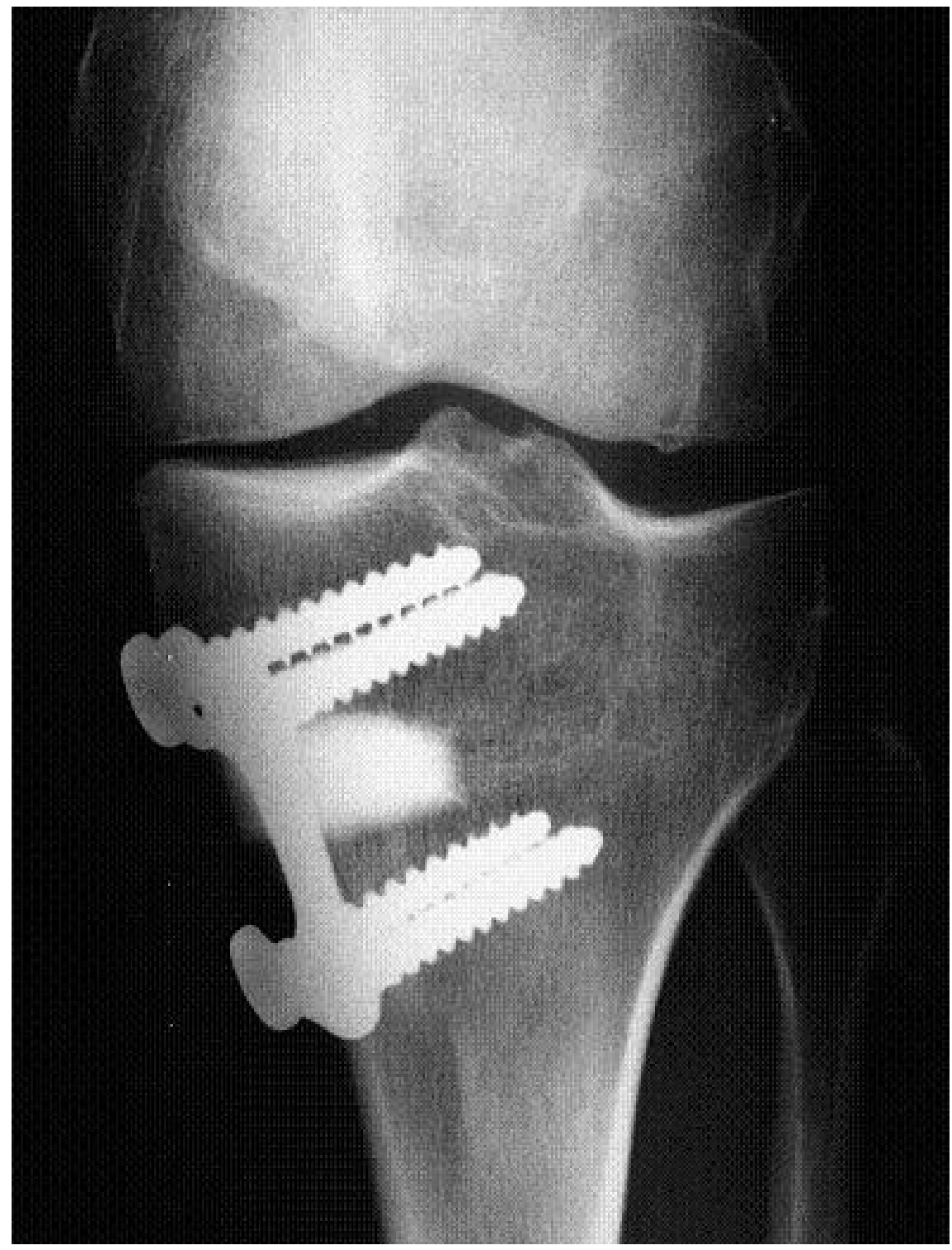

Fig. 10. c: Post Operative film of Computer navigated open wedge HTO showing correction of the varus deformity.

\subsection{Computer-assisted double level osteotomy (Fig.11 a,b,c)}

The first stage is essentially the same as for an HTO : percutaneous insertion of the rigid body markers (high enough not to hamper the femoral osteotomy and low enough on the other level to avoid interfering with the tibial osteotomy), followed by kinematic acquisition 
of the hip centre, middle of the knee and tibio-tarsal joints in order to find the mechanical axis of the lower limb.

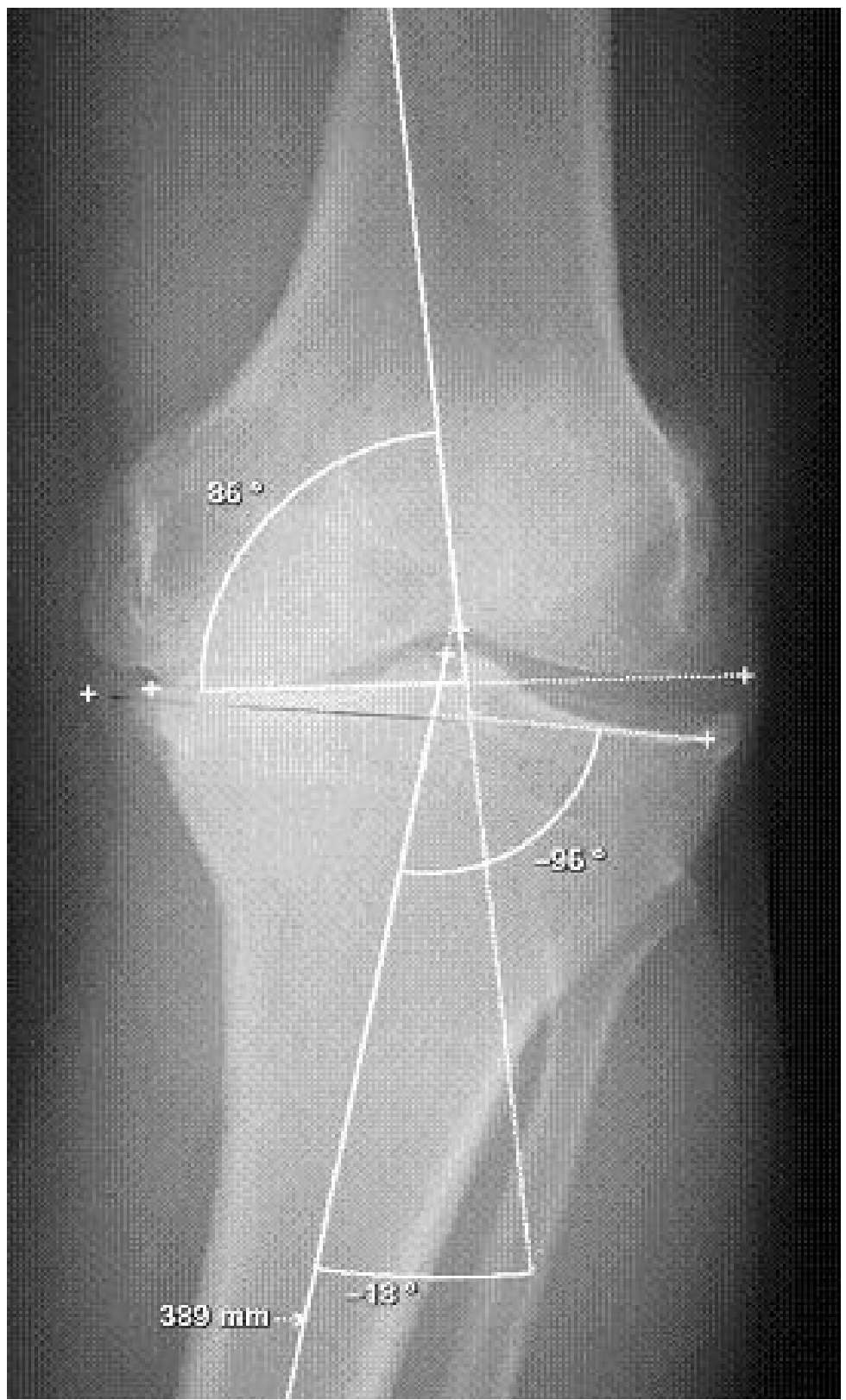

Fig. 11. a: A Pre-operative x-ray film of left knee, femoral and tibia varus deformity. Measurements show the degree of deformity in each bone needing to be corrected. 


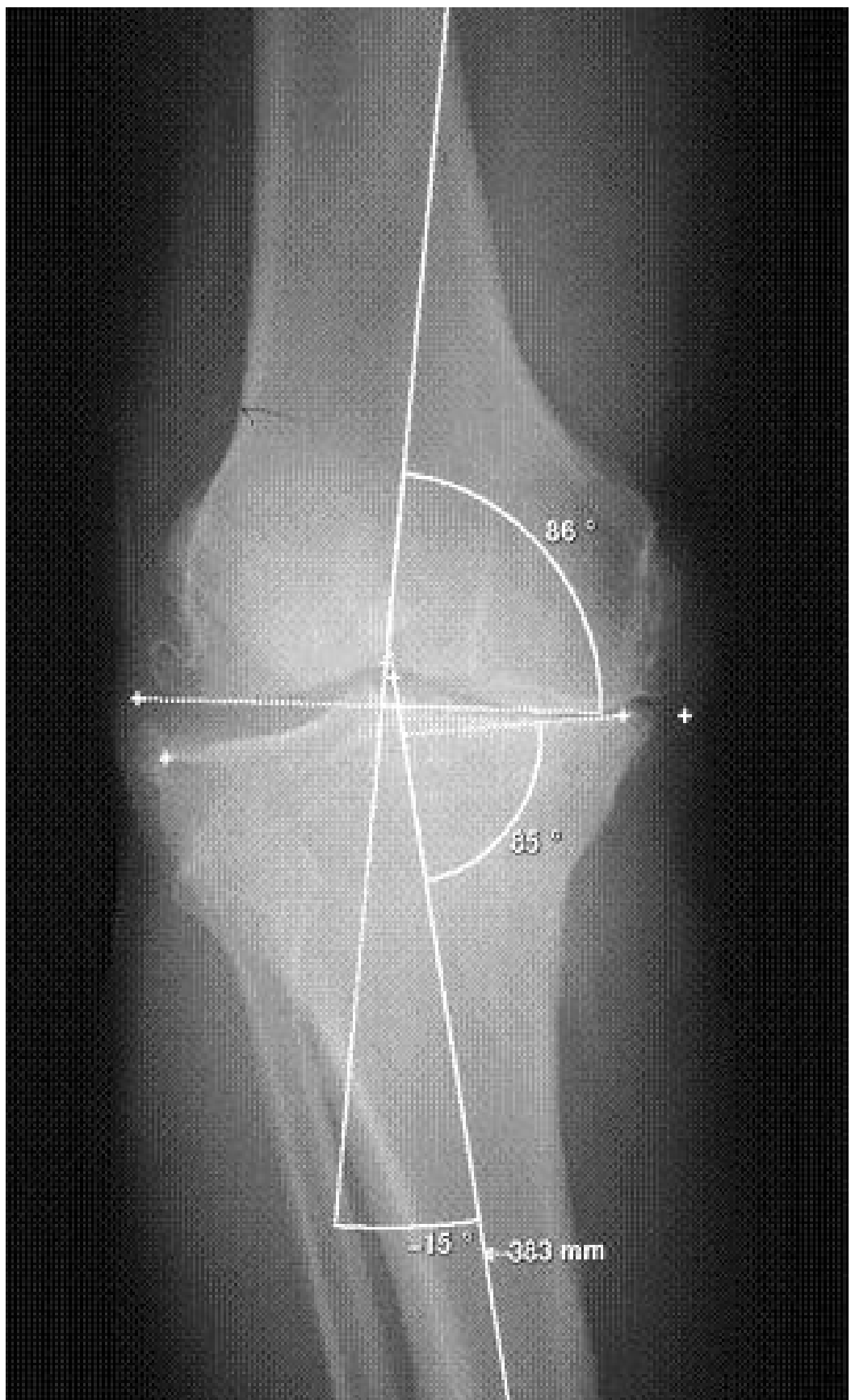

Fig. 11. b: Pre-op x-ray film of the right knee, femoral and tibia varus deformity. Measurements show the degree of deformity in each bone needing to be corrected. 


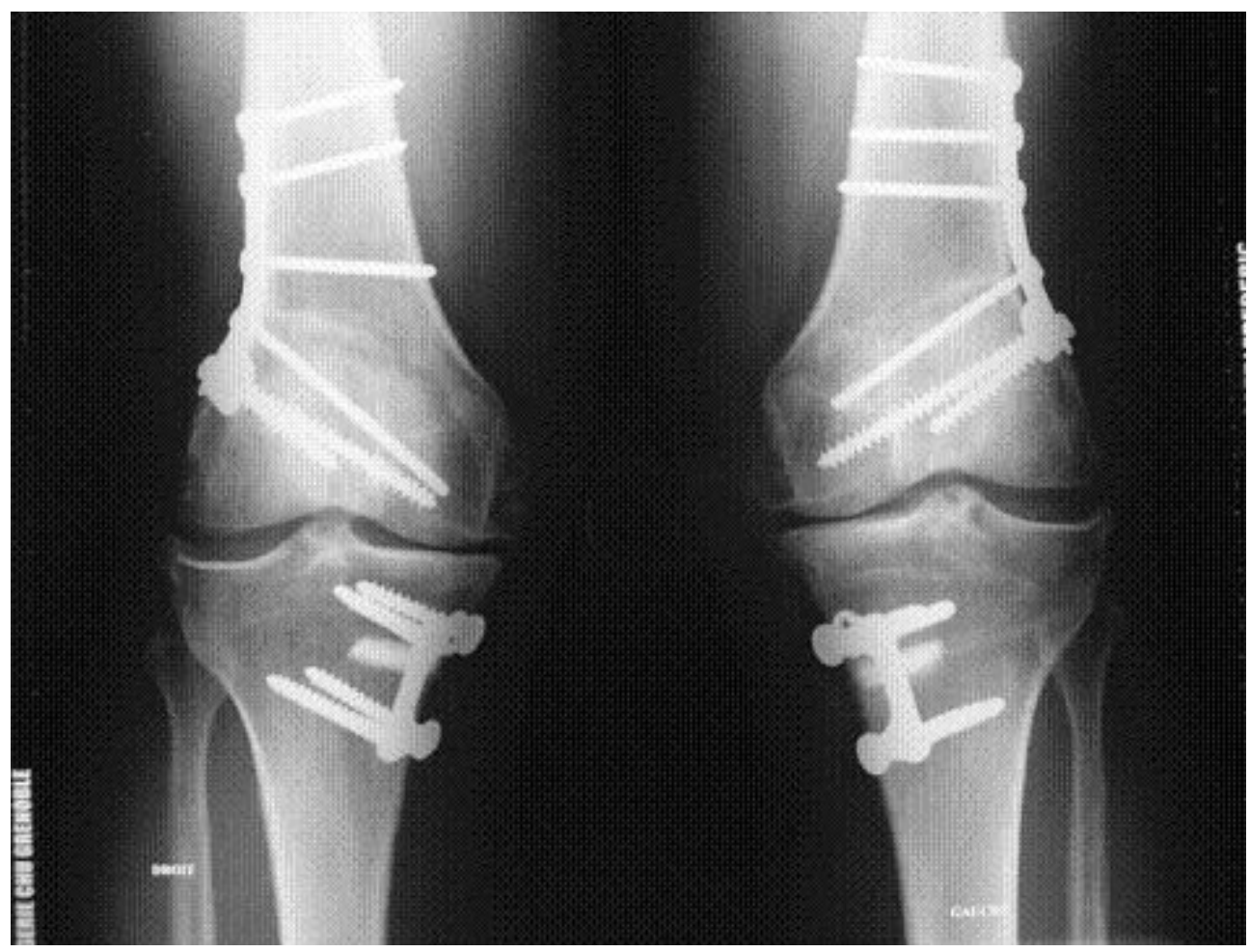

Fig. 11. c: Post operative Film of: Computer navigated double osteotomy for femoral tibia varus deformity. The deformity in both legs were corrected maintaining the joint line.

The second stage consists of making the femoral closing osteotomy in the distal femur (in general a $5-6^{\circ}$ alteration is made, although sometimes more in congenital femoral varus) and fixing it in position with a T-plate (AO/ Synthes). A lateral approach with elevation of the vastus lateralis is chosen, to allow the location of the proximal tip of the trochlea. The track of the osteotomy lies proximal to the trochlea and is directed obliquely from proximal lateral to distal medial femoral cortex (Fig.12). A wedge of bone is then excised from the distal femur with a $4-5 \mathrm{~mm}$ lateral base, corresponding to a $5-6^{\circ}$ correction (Fig.13). The osteotomy is then fixed with the T-plate after placing the femur into valgus manually (Fig. 14). Once this stage is reached the mechanical axis is rechecked so the required correction at the level of the tibia can be calculated in order to achieve the pre-operative objectives. Then the wound is closed on a drain. The last stage is to perform the HTO exactly in the fashion described earlier. The definitive axis is then displayed on the computer screen and the osteosynthesis is checked with the image intensifier.

\subsection{Computer-assisted distal femoral osteotomy}

The procedure is the same as described previously and we prefer to make a closing wedge osteotomy rather than an opening one because of the difficulty to get good stability after plating the distal femur. 


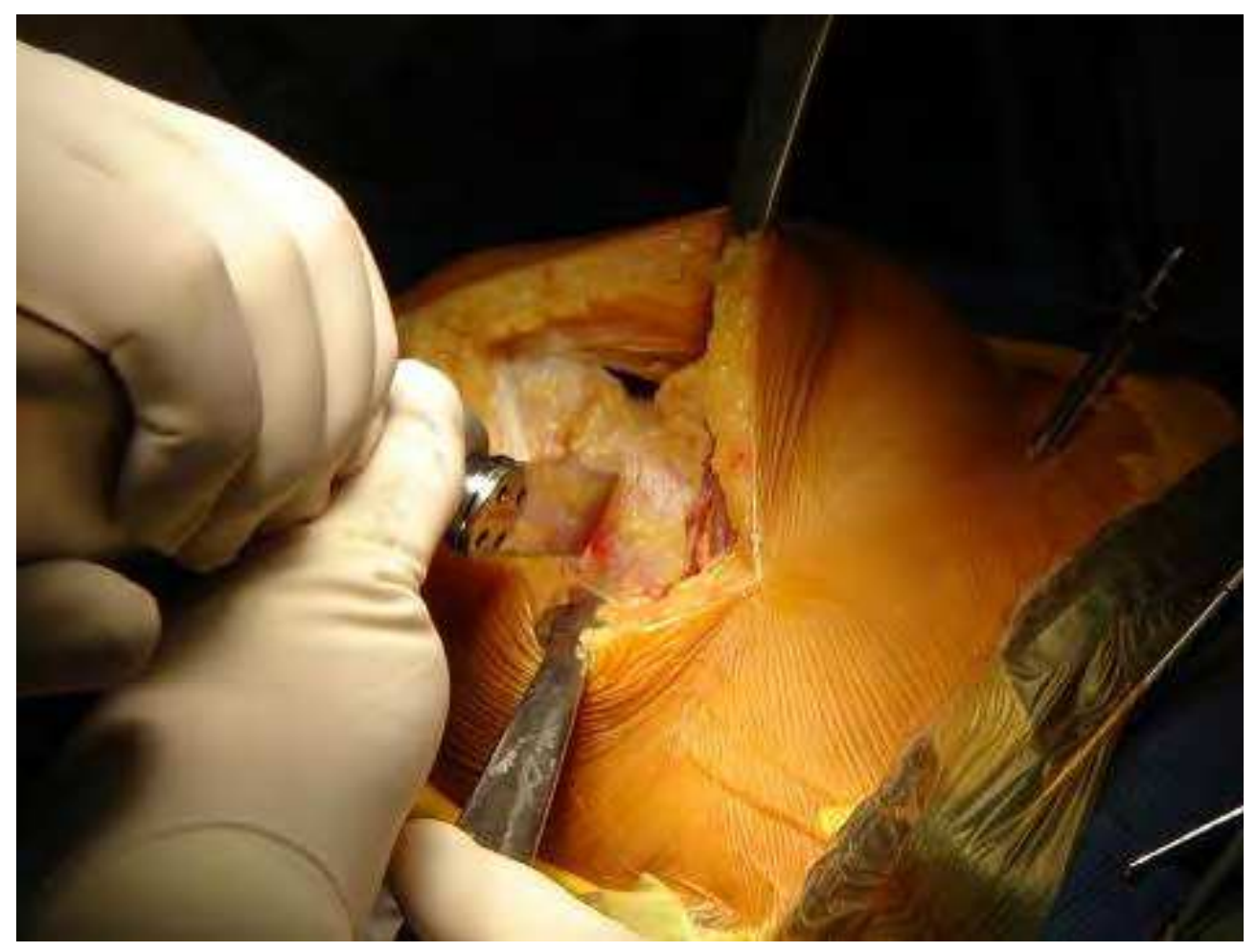

Fig. 12. Intra operation view of the direction of osteotomy proximal to trochlea aiming obliquely to distal medial femoral cortex. 


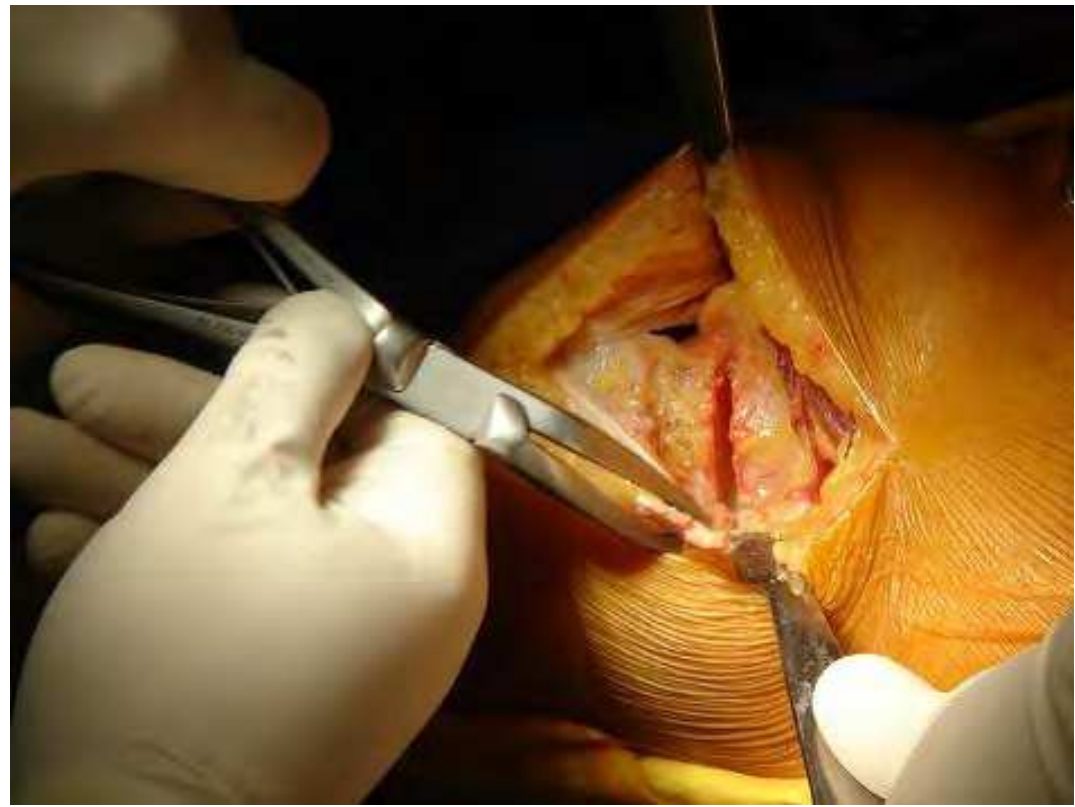

Fig. 13. Excision of 4-5 mm wedge of femur correspond to almost $5^{\circ}-6^{\circ}$ of deformity correction allowing 1-2 mm of saw play to avoid overcorrection.

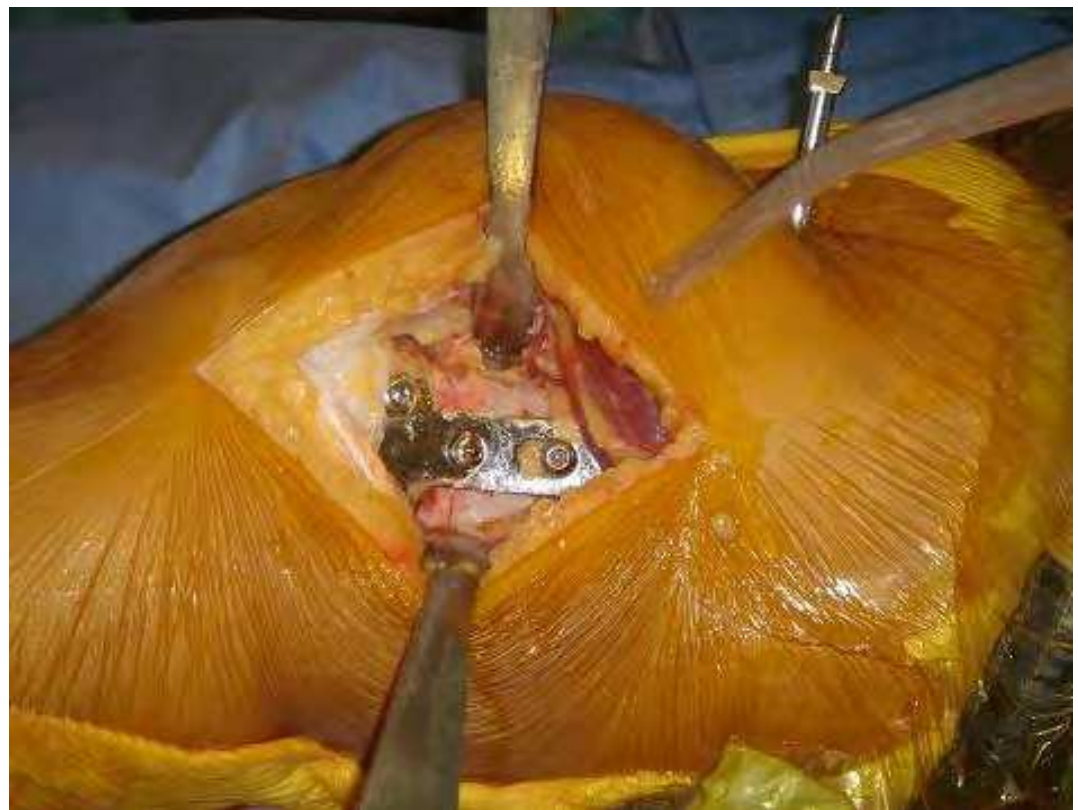

Fig. 14. Intra operation view showing correction of femoral varus deformity and maintaining it with a T-Plate. 


\section{Postoperative management}

The patient can stand up the day after the operation and walk with two crutches. Partial weight bearing is allowed for 4-6 weeks when performing an HTO and 10-12 weeks when performing DLO. Full range of motion is regained quickly after HTO and after 6-8 weeks for DLO, because of the distal femoral osteotomy which slows down rehabilitation. However, being an extra articular procedure, postoperative arthrofibrosis does not occur.

\section{Indications}

The best indication for osteotomy is a non-sedentary patient with a low arthritis grade (Coventry, MB \& Ilstrup, DM \& Wallrichs, SL 1993; Yasuda, K \& Majima, T \& Tsuchida, T et al 1992) and below 60 to 65 years. In some cases (very active patients under the age of 50 years) we have performed double level osteotomy for grade 4 and five with a good result but this is far from being the rule.

\section{Discussion}

When should double level osteotomy be performed? If we consider the "normal" mechanical axis of the lower limb as described by Kapandji (Kapandji, IA 1974) and later taken up by Hungerford and Krackow (Hungerford, DS \& Krackow, KA 1985) it should be $180^{\circ}$ with an MDFMA of $93^{\circ}$ and an MPTMA of $87^{\circ}$ resulting in a joint line perfectly parallel to the ground. However this assumption is not confirmed in case of osteoarthritis with varus deformity because, in unpublished series of senior author (D.S.) of 89 TKR, we found an MDFMA of $93^{\circ}$ in only $43.8 \%$ of the cases. It was at $90^{\circ}$ in $33.7 \%$ of the cases, below $90^{\circ}$ in $13.5 \%$, and above $93^{\circ}$ in $9 \%$.

Thus, before performing high tibial osteotomy, it is crucial to have high quality and reproducible full-length AP radiographs of the lower limb, according to a specific protocol. The HKA angle, the MDFMA and the MPTMA should be determined on this goniometry (Fig.3a and $3 \mathrm{~b}$ ). Lateral instability testing has become less important than it once was, being that since the indications for osteotomy in this setting have become rare. In case of femoral valgus (MDFMA $>90^{\circ}$ ), it is illogical to perform a femoral osteotomy because we do not want to create in the femur, the error, we are trying to avoid in the tibia. If the femur is in varus or at $90^{\circ}$, we think, we should proceed with a femoral osteotomy to achieve an MDFMA of around $93^{\circ}\left(93^{\circ}+/-2^{\circ}\right)$, and then complete it with a tibial osteotomy to achieve an HKA angle of $182^{\circ}+/-2^{\circ}$. In our experience, to overcorrect more than this, may jeopardize satisfactory results (Fig. 3c). Overcorrection, whether femoral or tibial, can distort the anatomy and lead to a much more complicated revision TKR. However we think a longer follow-up is needed to prove overcorrection by $+/-2^{\circ}$ is enough for a lasting good result. If the tibia is not in varus (MPTMA over $88^{\circ}$ ), we should perform a femoral osteotomy specially if the femur is at $90^{\circ}$ or in varus or contraindicate any osteotomy if it leads to joint line obliquity of more than $5^{\circ}$. If we strictly adhere to these criteria, indications for double level osteotomy will likely increase with the development of navigation systems, since as mentioned earlier, femurs in varus are not rare, and more so, those at $90^{\circ}$.

Combined distal femoral and proximal tibial osteotomy in the treatment of genu varum is technically difficult. Little has been said about this technique in the literature and we 
could find only one paper reporting on 24 patients (29 knees) operated on with a conventional technique (two closing wedge osteotomies) (Babis GC, An KN, Chao E. YS, et al, 2002). The mean preoperative HKA angle was $193.3^{\circ}$ (which is $13.3^{\circ}$ of varus) and they used a computer-aided analysis of the mechanical status of the knee for preoperative planning. This was limited to preoperative evaluation, and the reliability of the preoperative radiographic evaluation was not assessed. The results showed a mean postoperative HKA angle of $176.9^{\circ}\left(169.4^{\circ}\right.$ to $\left.184.9^{\circ}\right)$. They had a residual varus in 2 cases $\left(4.6^{\circ}\right.$ and $\left.4.9^{\circ}\right)$ and an over correction of more than $4^{\circ}$ in 10 cases and more than $6^{\circ}$ in 5 . One knows an under correction may lead to failure of the operative procedure and a too much overcorrection to discomfort.

The difficulty of the technique comes from the fact once the first osteotomy is performed, whether femoral or tibial, landmarks change and the ability to achieve a satisfactory alignment with the second osteotomy becomes challenging in the absence of reliable intraoperative landmarks. Martres et al (Martres, S \& Servien, E \& Aït Si Selmi, T, et al 2004) suggested performing this operation in two different stages to improve its accuracy and reproducibility. It is also justified to consider complication occurring at both osteotomy sites could lead to disastrous result. On the other hand, every surgeon operating on osteoarthritic knees should be aware of the risk of malunion in the proximal tibia, for a procedure often considered temporary, particularly when performing an isolated HTO. In fact every osteotomy in a young adult is susceptible to lead subsequently to a TKR, and thus it is essential to plan ahead for the iterative surgery called revision.

Computer-assisted surgery allows controlling of the femoro-tibial axis (HKA angle) at every step of the procedure and thus makes it more accurate. Our first results (Saragaglia, D \& Pradel, PH \& Picard, F 2004) showed in a comparative cohort study of computer-assisted versus conventional HTO, a $96 \%$ reproducibility in achieving a mechanical axis of $184^{\circ}+/-2^{\circ}$ in the computer navigated group versus $71 \%$ in the conventional osteotomy group $(\mathrm{p}<$ 0.0015). In another prospective series including 16 cases of DLO (Saragaglia, D \& RubensDuval, B \& Chaussard, C 2007) we showed $87.5 \%$ success in reaching our pre-operative goal for HKA angle, and $100 \%$ success in reaching the desired MPTMA $\left(90^{\circ}+/-2^{\circ}\right)$, which in terms of performance is remarkable. At the femoral level, results were less accurate $(75 \%$ of MDFMA at $95^{\circ}+/-2^{\circ}$ ). This could be related to the closing wedge osteotomy, which is less straight forward. Moreover, with the Orthopilot ${ }^{\circledR}$ device (kinematic model without pre-op imaging), controlling the MDFMA and the MPTMA following the two osteotomies is not possible because a large arthrotomy would be required to identify and palpate specific landmarks similar to the ones used for TKA.

Thus the only navigated parameter is the HKA angle; the others are calculated from pre and post-operative $\mathrm{X}$-Rays using the most rigorous planning. Regarding mid-term clinical and radiological results of DLO we reviewed recently 42 cases operated on between August 2001 and June 2010 that is with a mean follow up of $46+/-27$ months (12-108). The mean Lyshölm-Tegner score (Tegner, Y \& Lysholm, J \& Lysholm, M \& Gillquist, J 1986) improved from $41.2+/-8.9$ points (22-69) to $83.3+/-7.5$ points (62-91) and the KOOS score ( Roos, EM \& Roos, HP \& Ekdahl, C \& Lohmander, LS 1998) was 95.1+/- 3.2 points (89-100). 40 Patients were satisfied (22) or very satisfied (18) of the result. The radiological results showed the preoperative goal was reached in $92.7 \%$ of the cases for the HKA angle and in $88.1 \%$ for the 
MPTMA, with only one case over $93^{\circ}$. The mean HKA angle was $181.83^{\circ}+/-1.80^{\circ}\left(177^{\circ}\right.$ $\left.185^{\circ}\right)$, the mean MPTMA of $89.71^{\circ}+/-1.72^{\circ}\left(85^{\circ}-93^{\circ}\right)$ and the mean MDFMA of $92.76^{\circ}+/-$ $2.02^{\circ}\left(89^{\circ}-97^{\circ}\right)$. No patient was revised to total knee arthroplasty.

Finally, despite our trust in opening wedge osteotomies, we think, at the femoral level, one should perform a closing wedge osteotomy to avoid excessive lengthening of the limb when performing DLO (double opening) and also to avoid a less stable osteosynthesis.

\section{Conclusion}

Young patient genu varum deformity can be corrected by high tibial valgus osteotomy, but it is not the sole way to do. The indication is based on an accurate and reproducible radiological protocol including at least standing AP long leg X-ray. One must measure not only the HKA angle but also the medial distal femoral mechanical angle (MDFMA) and the medial proximal tibial mechanical angle (MPTMA). These measures will guide the surgeon to choose the best indication. When the MDFMA is in valgus $\left(93^{\circ}\right.$ or more) and the MPTMA in varus (below $88^{\circ}$ ) the best one is HTO. When the MDFMA is in varus ( $90^{\circ}$ or less) and the MPTMA in varus (below $88^{\circ}$ ) the best indication is DLO. Finally, when the MDFMA is in varus and the MPTMA above $88^{\circ}$ the best indication is DFO. This way of thinking should avoid too much oblique joint line, which is a difficult condition when performing revision to TKA.

\section{References}

Babis GC, An KN, Chao E.YS, et al (2002) Double level osteotomy of the knee :: a method to retain joint-line obliquity. J Bone J Surg Am 84 ::1380-13881.

Coventry MB, Ilstrup DM, Wallrichs SL (1993) Proximal tibial osteotomy :: a critical longterm study of eighty-seven cases. J Bone Joint Surg Am 75 ::196-201

Hakki S, Saleh K, Bilotta V, et al. (2009) Navigational predictors in determining the necessity for collateral ligament release in total knee replacement. JBJS - Br, Vol 91-B, Issue 9, 1178-1182.

Hernigou Ph, Medevielle D, Debeyre J, et al (1987) Proximal tibial osteotomy for osteoarthritis with varus deformity :: a ten to thirteen-year follow-up study. J Bone Joint Surg Am 69 ::332-354

Hungerford DS, Krackow KA (1985) Total joint arthroplasty of the knee. Clin Orthop 192 $:: 23-30$

Jackson JP, Waugh W (1961) Tibial osteotomy for osteoarthritis of the knee. J Bone Joint Surg Br $43:: 746-751$

Jenny JY, Tavan A, Jenny G, et al (1998) Taux de survie à long terme des ostéotomies tibiales de valgisation pour gonarthrose. Rev chir Orthop 84 ::350-357

Judet R, Dupuis JF, Honnard F, et al (1964) Désaxations et arthroses du genou. Le genu varum de l'adulte. Indications thérapeutiques, résultats. Rev Chir Orthop 13 ::128

Kapandji IA (1974) Physiologie articulaire . Fascicule II quatrième édition :: membre inférieur. Paris , PA :: Maloine SA ; ::104 
Lerat JL (2000) Ostéotomies dans la gonarthrose. Cahiers d'enseignement de la SOFCOT 2000. Paris, PA :: Elsevier ; ::165-201

Lootvoet L, Massinon A, Rossillon R, et al (1993) Ostéotomie tibiale haute de valgisation pour gonarthrose sur genu varum :: à propos d'une série de 193 cas revus après 6 à 10 ans de recul. Rev Chir Orthop 79 ::375-384

Martres S, Servien E, Aït Si Selmi T, et al (2004) Double ostéotomie :: indication dans la gonarthrose. Rev Chir Orthop 90 suppl au nº :: 2S137-2S138

Merle d'Aubigné R, Ramadier JO (1961) Arthrose du genou et surcharge articulaire. Acta Orthop Belg $27:: 365-375$

Papachristou G, Plessas S, Sourlas J, Levidiotis C, Chronopoulos E, Papachristou C (2006). Deterioration of long-term results following high tibial osteotomy in patients under 60 years of age. Int orthop 30: 406-408

Picard F, Leitner F, Raoult A, et al (1999) Computer assisted knee arthroplasty. In " Reschnergestützte Verfahren in Orthopädie und Unfallchirurgie ». Steinkopff Darmstadt, PA :: Jerosch, Nichol and Peikenkam; :: 461-471.

Ramadier JO, Buard JE, Lortat-Jacob A, et al (1982) Mesure radiologique des déformations frontales du genou. Procédé du profil vrai radiologique. Rev Chir Orthop 68 :7578

Rinonapoli E, Mancini GB, Corvaglia A, et al (1998) Tibial osteotomy for varus gonarthrosis. A 0 to 21-year follow-up study. Clin Orthop $353::$ 185-193

Roos EM, Roos HP, Ekdahl C, Lohmander LS (1998) Knee Injury and Osteoarthritis Outcome Score (KOOS): validation of a swedish version. Scand J Med Sci Sports 8: $439-48$

Saragaglia D, Blaysat M, Inman D, Mercier N (2010) Outcome of opening wedge High tibial osteotomy augmented with a Biosorb wedge and fixed by a plate with a mean of ten years follow up. Int Orthop. Published on line in August 2010 (DOI 10.1007/s00264-010-1102-9)

Saragaglia D, Pradel Ph, Picard F (2004) L'ostéotomie de valgisation assistée par ordinateur dans le genu varum arthrosique :: résultats radiologiques d'une étude cas-témoin de 56 cas. E-mémoires de l'Académie Nationale de Chirurgie 3 :: 21-25. Available at :: http://www.bium.univ-paris5.fr/acad-chirurgie.

Saragaglia D, Picard F, Chaussard C, et al (2001) Mise en place des prothèses totales du genou assistée par ordinateur :: comparaison avec la technique conventionnelle. Résultats d'une étude prospective randomisée de 50 cas. Rev chir Orthop 87 ::1828

Saragaglia D, Roberts J (2005) Navigated osteotomies around the knee in 170 patients with osteoarthritis secondary to genu varum. Orthopaedics 28 Suppl nº 10 ::S1269 S1274

Saragaglia D, Rubens-Duval B, Chaussard C (2007) Double ostéotomie assistée par ordinateur dans les grands genu varum. Résultats préliminaires à propos de 16 cas. Rev Chir Orthop 93::351-356

Tegner Y, Lysholm J, Lysholm M, Gillquist J (1986) A performance test to monitor rehabilitation and evaluate anterior cruciate ligament injuries. Am J Sports Med 14 : 156-159 
Yasuda K, Majima T, Tsuchida T, et al (1992) A ten to 15 year follow-up observation of high tibial osteotomy in medial compartment osteoarthrosis. Clin Orthop 282 ::186-195 


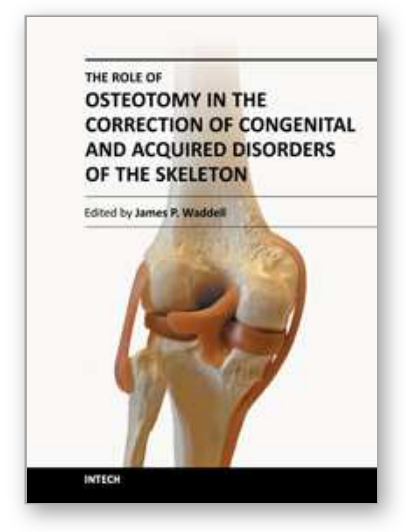

\author{
The Role of Osteotomy in the Correction of Congenital and \\ Acquired Disorders of the Skeleton \\ Edited by Prof. James Waddell
}

ISBN 978-953-51-0495-7

Hard cover, 294 pages

Publisher InTech

Published online 11, April, 2012

Published in print edition April, 2012

This book demonstrates specific osteotomy techniques from the skull to the hallux. The role of osteotomy in the correction of deformity is under appreciated in part because of the ubiquitous nature of joint replacement surgery. It should be remembered, however, that osteotomy has a role to play in the correction of deformity in the growing child, the active young adult, and patients of any age with post-traumatic deformity limiting function and enjoyment of life. In this text we bring you a number of papers defining specific problems for which osteotomy is found to be an effective and lasting solution. I hope you find it useful.

\title{
How to reference
}

In order to correctly reference this scholarly work, feel free to copy and paste the following:

Dominique Saragaglia and Sam Hakki (2012). Computer-Assisted High Tibial and Double Level Osteotomies for Genu Varum Deformity, The Role of Osteotomy in the Correction of Congenital and Acquired Disorders of the Skeleton, Prof. James Waddell (Ed.), ISBN: 978-953-51-0495-7, InTech, Available from:

http://www.intechopen.com/books/the-role-of-osteotomy-in-the-correction-of-congenital-and-acquireddisorders-of-the-skeleton/computer-assisted-high-tibial-and-double-level-osteotomies-for-genu-varumdeformity

\section{INTECH}

open science | open minds

\section{InTech Europe}

University Campus STeP Ri

Slavka Krautzeka 83/A

51000 Rijeka, Croatia

Phone: +385 (51) 770447

Fax: +385 (51) 686166

www.intechopen.com

\section{InTech China}

Unit 405, Office Block, Hotel Equatorial Shanghai

No.65, Yan An Road (West), Shanghai, 200040, China 中国上海市延安西路65号上海国际贵都大饭店办公楼 405 单元

Phone: +86-21-62489820

Fax: +86-21-62489821 
(C) 2012 The Author(s). Licensee IntechOpen. This is an open access article distributed under the terms of the Creative Commons Attribution 3.0 License, which permits unrestricted use, distribution, and reproduction in any medium, provided the original work is properly cited. 